REVESCO. Revista de Estudios Cooperativos

ISSN: $1885-8031$

http://dx.doi.org/10.5209/REVE.60989

\title{
Aproximación al perfil y calidad del empleo generado por las sociedades cooperativas andaluzas
}

\author{
María del Carmen Pérez González ${ }^{1}$ y Lidia Valiente Palma²
}

Recibido: 13 de febrero de 2018 / Aceptado: 10 de julio de 2018

Resumen. El objetivo del presente trabajo ha consistido en identificar y caracterizar el perfil y la calidad del empleo en las cooperativas en Andalucía en comparación con el resto del tejido empresarial, con el fin de determinar si verdaderamente presentan una mayor calidad frente al resto de empresas. Las cooperativas, organizaciones de Economía Social que destacan en la región andaluza en términos cuantitativos, se caracterizan por ser empresas que actúan bajo principios y valores sociales, por lo que identificar qué tipo de empleo existe en estas organizaciones es de especial interés en cuanto al establecimiento de políticas específicas territoriales. Para alcanzar el objetivo planteado, se han realizado análisis descriptivos y pruebas no paramétricas utilizando la Muestra Continua de Vidas Labores (MCVL) del Ministerio de Empleo y Seguridad Social español. Entre los resultados obtenidos destaca que estas organizaciones pueden presentarse como oportunidad de empleo, en mayor grado que el resto de empresas mercantiles o convencionales, para colectivos sociales con dificultades de acceso al mercado laboral, como son los jóvenes y los mayores de 45 años de edad. Esto podría justificar el hecho de conceder a las cooperativas un papel significativo en las estrategias y políticas públicas que fomenten el empleo en Andalucía.

Palabras clave: Cooperativas; Andalucía; Muestra Continua de Vidas Laborales (MCVL); Empresas mercantiles; Empleo; Pruebas no paramétricas.

Claves Econlit: A13; J16; J40; J54.

\section{[en] Approximation to the profile and quality of the employment generated by the andalusian cooperative societies}

\begin{abstract}
The aim of this study has been to identify and characterize the profile and quality of employment generated by cooperative societies in Andalusia in comparison to the traditional business sector, in order to determine whether really they have a higher quality over all companies. The cooperatives are entities of Social Economy which base their operations on a series of social, economic and environmental principles and values. In addition, a significant weight of these societies exists in this region. Therefore, identifying what type of employment exists in these organizations is of special interest for the establishment of specific territorial policies. In order to achieve the objective proposed, descriptive analysis and non parametric tests with the Continuous Professional Life Sample of the Ministry of Employment and Social Security of Spain has been performed. The cooperatives as an opportunity of employment to a greater degree than the traditional business sector for certain social
\end{abstract}

1 Universidad de Cádiz, España

Dirección de correo electrónico: maricarmen.perez@uca.es

2 Universidad de Cádiz, España

Dirección de correo electrónico: valientepalma@gmail.com 
groups who have difficulties of access to the labour market such as young people and over the age of 45 is among the main results. In this way, granting a significant role to cooperative societies of the public strategies and policies in Andalusia would be justified.

Keywords: Cooperatives; Andalusia; Continuous Professional Life Sample; Business enterprises; Employment; Non parametric tests.

Sumario. 1. Introducción. 2. Metodología y datos empleados. 3. Resultados. 4. Consideraciones finales. 5. Referencias bibliográficas.

Cómo citar: Pérez González, M.C. y Valiente Palma, L. (2019) Aproximación al perfil y calidad del empleo generado por las sociedades cooperativas andaluzas. REVESCO. Revista de Estudios Cooperativos, Primer Cuatrimestre, No 130, pp. 122-148. DOI: 10.5209/REVE.60989.

\section{Introducción}

La última crisis, iniciada a finales de 2007, ha desencadenado una serie de consecuencias socioeconómicas desfavorables, manifestadas éstas principalmente en las altas tasas de desempleo alcanzándose, como punto álgido, casi el $27 \%$ en el primer trimestre de 2013 en España, según datos de la Encuesta de Población Activa (EPA) $)^{3}$ Pero estas cifras son aún más elevadas en determinadas regiones españolas como en el caso de Andalucía, comunidad autónoma en la que se alcanzó, para el mismo periodo, el 36,77\%. Datos aún más preocupantes son las tasas de paro de determinados colectivos sociales como jóvenes y mujeres que en la actualidad ${ }^{4}$ superan los valores del $48 \%$ y $28,84 \%$ respectivamente, muy superiores a la media nacional (los jóvenes menores de 25 años presentan una tasa del $34 \%$, mientras que la de mujeres se sitúa en el $18 \%$ ).

Por otro lado, en Andalucía existe cierto reconocimiento institucional por las organizaciones de Economía Social (en adelante ES), que se ha manifestado a partir de diferentes acciones como las leyes reguladoras de sus entidades, los Pactos Andaluces por la Economía Social o los diferentes programas de apoyo a sus organizaciones. Además, atendiendo a las sociedades cooperativas, las entidades más representativas de la ES en España, hay que destacar que a finales del año 2016 existían en Andalucía 3.994 que daban empleo a 57.864 personas, según datos del Ministerio de Empleo y Seguridad Social (MEYSS). Esto supone que el $19 \%$ de las cooperativas y el $18 \%$ del empleo generado por éstas en el territorio español, se encuentra en Andalucía. Estas organizaciones son definidas por la Alianza Cooperativa Internacional (ACI) como "asociaciones de personas que se unen voluntariamente para satisfacer sus necesidades y aspiraciones económicas, sociales y culturales comunes, por medio de una empresa de propiedad conjunta gestionada democráticamente"5. A ello hay que añadir que basan su funcionamiento en una serie de principios y valores de actuación, como son el de "educación, formación e información" de sus miembros o el de "interés por la comunidad" (ACI, 1995), anteponiendo, de esta forma, la persona al capital

3 En la actualidad la tasa de paro en España se sitúa en el 16,38\% (datos para el tercer trimestre de 2017, según la EPA).

4 Datos según la Encuesta de Población Activa para el tercer trimestre de 2017.

5 https://ica.coop/en/what-co-operative. 
social y actuando mediante criterios de responsabilidad interna y externa (Monzón, 2006; Barea, 2008).

Estos principios y valores de las cooperativas en particular (y de la ES en general), pueden hacer que se planteen como fórmulas idóneas ante determinadas dificultades socioeconómicas. Las cooperativas promueven el asociacionismo mediante el autoempleo colectivo, adquiriendo, sus miembros, la condición de socio-trabajador por su participación activa en los procesos productivos y en la toma de decisiones (García-Gutiérrez et al., 2013; Lejarriaga, Bel y Martín, 2013) mediante una gestión democrática que, a su vez, traslada los valores al ámbito económico (Dívar, 2011). A ello hay que añadir el desarrollo de trabajos que han demostrado la mayor resistencia de estas organizaciones frente a variaciones del ciclo económico, en concreto en cuanto a la destrucción de empleo ante fases de recesión económica y que, siguiendo a Calderón y Calderón (2012a), son tanto de autores de carácter nacional (Tomás-Carpi, 1997; Grávalos y Pomares, 2001; Díaz y Marcuello, 2010) como internacionales Guerreri, Nazzaro y Zevi, 1992; Cornforth y Thomas, 1995; Informe CECOP-CICOPA, 2011).

Por ello, la existencia de un número significativo de sociedades cooperativas en la comunidad autónoma de Andalucía, se convierte en un potencial u oportunidad para fomentar empleo estable y calidad dado el contexto socioeconómico actual. No obstante, previamente, es necesario analizar, qué tipo de empleo están generando más allá de determinar su resistencia ante variaciones en el ciclo económico. Es decir, ¿se puede afirmar que existe determinada calidad en el empleo que generan las cooperativas?

En lo que se refiere al concepto de calidad del empleo, se parte del establecido por la Comisión Europea $(2001)^{6}$ que determina dos grandes grupos para recoger las diversas dimensiones que dicho concepto presenta (Comisión Europea, 2001: 8):

1. Las características de los puestos de trabajo: la satisfacción profesional, la remuneración, la jornada laboral, las cualificaciones, la formación y las perspectivas de trayectoria profesional o el contenido del trabajo, entre otras.

2. El entorno de trabajo y el mercado laboral: entre las que se encuentra la igualdad entre hombres y mujeres, la flexibilidad y seguridad, la inclusión y el acceso al mercado laboral, la conciliación entre la vida profesional y la vida privada, el diálogo social y participación de los trabajadores o la diversidad y no discriminación.

En el presente trabajo se incide en los dos grupos: por un lado, se trata la jornada laboral y la formación y cualificación de los trabajadores como características de los puestos de trabajo (a partir del tipo de contrato, la jornada laboral y el grupo de cotización de los trabajadores) y, por otro, la igualdad entre hombres y mujeres (incluyendo la variable sexo), la flexibilidad y seguridad (mediante los tipos de contrato y jornada laboral) (Calderón y Calderón, 2012b) y el acceso al mercado laboral (a partir del número de trabajadores por edades) como características del entorno de trabajo. A partir de estas variables, se establece una

Comunicación de la Comisión al Consejo, al Parlamento Europeo, al CESE y al Comité de las Regiones, de 20 de junio de 2001 [COM (2001) 313 final]. 
aproximación al perfil y calidad del empleo generado por las sociedades cooperativas de Andalucía.

En concreto, y en línea con lo anterior, el objetivo de este trabajo ha consistido en identificar y caracterizar el perfil y la calidad del empleo en las sociedades cooperativas andaluzas en comparación con el resto del tejido empresarial, representado éste último por las empresas mercantiles (sociedades anónimas y sociedades limitadas), con el fin de determinar si verdaderamente presentan una mayor calidad frente al del resto de empresas, lo que podría justificar el concederles un significativo papel en las estrategias y políticas públicas encaminadas a mantener o impulsar la generación de empleo en la región.

Para ello, la estructura seguida en el presente trabajo consiste en, tras esta introducción, un apartado donde se detalla la metodología aplicada en la investigación, un segundo epígrafe donde se presentan los resultados y su discusión $\mathrm{y}$, finalmente, un tercer apartado que recoge las consideraciones finales derivadas del presente estudio. Entre éstas últimas se encuentra que las sociedades cooperativas pueden presentarse como oportunidad o alternativa para paliar las dificultades con las que cuentan determinados colectivos sociales, como son los jóvenes y mayores de 45 años de edad, para incorporarse al mercado laboral o lograr puestos de trabajo más estables.

El presente trabajo supone un valor añadido a trabajos previos citados sobre las ventajas que suponen las sociedades cooperativas frente al resto de organizaciones convencionales o sector empresarial tradicional, desde la perspectiva del empleo, centrándose esta aportación en la comunidad autónoma de Andalucía y determinando el perfil y la calidad de dicho empleo. Ello contribuye a un mayor conocimiento del mercado laboral relacionado con estas empresas que se traduce en la posibilidad de diseñar políticas específicas en torno a las mismas, con mayor garantía de éxito.

\section{Metodología y datos empleados}

Para determinar el perfil y la calidad del empleo, desde un análisis comparado, se han desarrollado análisis descriptivos sobre los trabajadores en estas organizaciones, sociedades cooperativas y sociedades mercantiles, por sexo, edad, nivel educativo, grupo de cotización, tipo de contrato y actividades económicas, a partir de tablas de contingencias realizadas para tal fin. Además, este análisis descriptivo se ha complementado con test de chi-cuadrado de Pearson $\left(\chi^{2}\right)$ y de corrección de continuidad de Yates aplicado a los primeros cuando las tablas de contingencia para las variables han sido de $2 \times 2$. Estas pruebas permiten comprobar si dos variables cualitativas están o no asociadas, bajo la hipótesis nula de la inexistencia de relación entre éstas.

Para el test de chi-cuadrado $\left(\chi^{2}\right)$ el estadístico de contraste para una tabla de contingencia de $r$ filas y $k$ columnas es el siguiente:

$$
\chi^{2}=\sum_{i=1}^{r} \sum_{j=1}^{k} \frac{\left(o_{i j}-E_{i j}\right)^{2}}{E_{i j}}, \text { con }(r-1)(k-1) \text { grados de libertad, }
$$


donde $O_{i j}$ representa la frecuencia observada en la fila $i$ de la columna $j$ y $E_{i i}$ la frecuencia esperada en caso de independencia entre las variables. De esta forma, el estadístico $\chi^{2}$ mide la diferencia entre el valor esperado -el que correspondería si las variables fuesen independientes- y el observado. A mayor diferencia entre ambos, mayor relación existirá entre las variables objeto de análisis. Cuando las tablas de contingencia son de $2 \times 2$ solo existe un grado de libertad por lo que el estadístico chi-cuadrado estará sobreestimado. Por ello debe aplicarse un factor de corrección conocido como la corrección de Yates para la continuidad, ya que la distribución chi-cuadrado es continua y se está aplicando a fenómenos discretos. Lo anterior se corrige con la siguiente expresión matemática:

$$
\chi_{Y}^{2}=\sum_{i=1}^{r} \sum_{j=1}^{k} \frac{\left(\left|o_{i j}-E_{i j}\right|-0,5\right)^{2}}{E_{i j}}
$$

En lo que se refiere a los datos para llevar a cabo el análisis anterior, se han utilizado los correspondientes a la Muestra Continua de Vidas Laborales (MCVL), en su versión con datos fiscales, para el año 2015, del Ministerio de Empleo y Seguridad Social. De manera general, esta muestra ha sido previamente utilizada para identificar la evolución laboral de los trabajadores en un determinado puesto (Cebrián y Toharia, 2008), determinar las diferencias salariales entre indefinidos (Clemente, García y Sanso, 2008), establecer el gasto que generan las pensiones contributivas del sistema de seguridad social español (Moral-Arce, Patxot y Souto, 2008) o para definir a la población inmigrante que cotiza al sistema público de pensiones (Domínguez-Fabián y Encinas-Goenechea, 2008).

Particularmente, en lo que respecta a las sociedades cooperativas, estos datos de la MCVL se han tomado en trabajos previos para diferentes finalidades: para medir la estabilidad del empleo en periodos de crisis (Calderón y Calderón, 2012a), determinar las diferencias en las condiciones laborales desde una perspectiva de género (Santero y Castro, 2016), identificar los principales beneficios en términos de utilidad social por parte de estas organizaciones (Martínez, Arcas y García, 2011) o determinar las diferencias salariales entre los trabajadores de cooperativas y trabajadores de empresas convencionales (Clemente et al., 2012).

Esta muestra está formada por microdatos, información de personas con carácter anónimo, que han tenido algún tipo de relación con la seguridad social en el año de referencia de la muestra. Para elaborar las MCVL, se selecciona el $4 \%$ de todas las personas registradas en el territorio español mediante un muestreo aleatorio simple. En lo que respecta a su estructura, la información se agrupa en seis tablas que recoge información sobre la persona (tabla 1), vida laboral o afiliación (tabla 2), bases de cotización (tabla 3), pensiones (tabla 4), convivientes (tabla 5) y datos fiscales (tabla 6), que provienen de las bases de datos de la Seguridad Social, del Padrón Continuo Municipal del INE y de las Retenciones e Ingresos a cuenta de la Agencia Estatal Tributaria española. No obstante, en este caso, se ha considerado la información referente a la tabla de personas y de afiliaciones o vida laboral, vinculando la información de ambas mediante un número de identificación de la persona física que figura en todas las tablas.

Para el año 2015, esta muestra contiene 22.381.074 registros de afiliaciones correspondientes a 1.194.837 personas. La cifra de afiliaciones es elevada debido a 
que para cada persona se incluye información del año de referencia, así como de años anteriores, es decir, su vida laboral.

A partir de esta muestra, se han realizado varias filtraciones de estos datos: en primer lugar, de la tabla de afiliaciones, mediante la variable domicilio de actividad de la cuenta de cotización, se han seleccionado las de la comunidad autónoma de Andalucía. En segundo lugar, se han eliminado aquellas relaciones con la seguridad social que no implican empleo en el año 2015, así como aquellos grupos de cotización para los que el dato no consta. En tercer y último lugar, se ha seleccionado de la muestra las afiliaciones que pertenecen a sociedades cooperativas, por un lado, y las que pertenecen a empresas mercantiles, es decir sociedades anónimas y sociedades limitadas, por otro.

Una vez realizadas las filtraciones anteriores, las muestras resultantes son las siguientes: 7.717 afiliaciones para las cooperativas y 142.755 para las empresas mercantiles. A partir de esta filtración, se ha trabajado con una serie de variables de interés de cada una de las tablas seleccionadas: de la tabla personas de la MCVL se ha trabajado con las variables fecha de nacimiento, sexo y nivel educativo; y de la de afiliaciones, con régimen de cotización, grupo de cotización, tipo de contrato de trabajo, coeficiente de tiempo parcial y actividad económica de la cuenta de cotización (CNAE, 2009).

\section{Resultados}

\subsection{Empleo desde la perspectiva de género: tipo de contrato y jornada laboral}

Analizando las afiliaciones a la Seguridad Social resultantes una vez realizadas las operaciones de filtración en la muestra inicial, hay que señalar que dichas afiliaciones corresponden a un total de 4.657 personas para el caso de las sociedades cooperativas y de 68.024 para las empresas mercantiles, siendo las afiliaciones medias de las segundas superiores (con un valor de 2,10 afiliaciones por personas frente a 1,66 de los trabajadores en cooperativas ${ }^{7}$. Esto, a priori, puede ser un indicio de que en las empresas convencionales la duración de los contratos es menor o existe una mayor temporalidad, es decir, existe menor estabilidad laboral en éstas (Tabla 1).

Sin embargo, en lo que respecta a los tipos de contratos, indefinidos o temporales, por cada contrato indefinido existen 3,68 temporales en las cooperativas, mientras que este ratio para las sociedades mercantiles es de 3 temporales por cada indefinido.

Atendiendo tanto al número de trabajadores como al de afiliaciones de éstos, por tipos de contrato y sexo, hay que destacar lo siguiente: existe una mayor proporción de mujeres que de hombres en las cooperativas (51\% y $49 \%$ respectivamente), representando a su vez, las mujeres, un 51\% de las afiliaciones frente al 49\% de los hombres); por su parte, en las empresas mercantiles se dan valores inferiores, contando con un $42 \%$ de mujeres que representan el $41 \%$ del

\footnotetext{
Un mismo trabajador puede presentar varias afiliaciones a la Seguridad Social en el año objeto de estudio, lo que indica determinada inestabilidad en un determinado puesto de trabajo.
} 
total de las afiliaciones, siendo, por tanto, los valores para los hombres $(58 \%)$ superiores que en el caso de las cooperativas. Además, las afiliaciones medias de las mujeres en empresas cooperativas $(1,64)$ cuenta con un valor inferior que el de empresas convencionales $(2,06)$ (Tabla 1$)$, poniendo de manifiesto un mayor grado de estabilidad laboral en estas empresas de ES.

Tabla. 1. Afiliaciones en la MCVL que implican situación de empleo

\begin{tabular}{|c|c|c|c|c|c|c|c|c|c|c|}
\hline \multirow[b]{3}{*}{ Afiliaciones } & \multicolumn{5}{|c|}{ Cooperativas } & \multicolumn{5}{|c|}{ Empresas mercantiles } \\
\hline & \multicolumn{2}{|c|}{ Homb. } & \multicolumn{2}{|l|}{ Muj. } & \multirow{2}{*}{$\begin{array}{l}\text { Tot. } \\
7.717\end{array}$} & \multicolumn{2}{|l|}{ Homb. } & \multicolumn{2}{|l|}{ Muj. } & \multirow{2}{*}{$\frac{\text { Tot. }}{142.755}$} \\
\hline & 3.786 & $49 \%$ & 3.931 & $51 \%$ & & 84.514 & $59 \%$ & 58.241 & $41 \%$ & \\
\hline Personas & 2.260 & $49 \%$ & 2.397 & $51 \%$ & 4.657 & 39.692 & $58 \%$ & 28.332 & $42 \%$ & 68.024 \\
\hline $\begin{array}{l}\text { Afiliac.medias por } \\
\text { persona }\end{array}$ & 1,68 & & 1,64 & & 1,66 & 2,13 & & 2,06 & & 2,10 \\
\hline \multicolumn{11}{|c|}{ Afiliaciones por tipo de contrato: } \\
\hline Indefinidos & 742 & $50 \%$ & 731 & $50 \%$ & 1.473 & 19.630 & $55 \%$ & 15.874 & $45 \%$ & 35.504 \\
\hline Temporales & 2.556 & $47 \%$ & 2.876 & $53 \%$ & 5.432 & 64.357 & $60 \%$ & 42.097 & $40 \%$ & 106.454 \\
\hline Jubilación parcial & 11 & & 3 & & 14 & 17 & & 10 & & 27 \\
\hline No constan & 477 & & 321 & & 798 & 297 & & 220 & & 517 \\
\hline
\end{tabular}

Fuente: Elaboración propia a partir de la MCVL 2015

Este mayor número de mujeres que de hombres en las sociedades cooperativas se justifica por lo siguiente: para contratos indefinidos, en el caso de las cooperativas, el porcentaje sobre el total se iguala entre hombre y mujeres, es decir, alrededor del $50 \%$ de estos trabajadores con carácter indefinido son mujeres, no ocurriendo lo mismo para los temporales, en el que éstas representan el 53\% superando así a los hombres. En las empresas mercantiles, los hombres tienen mayor proporción tanto en los contratos indefinidos como temporales, con un 55\% y $60 \%$ respectivamente (Tabla 1 ).

En línea con lo anterior, para determinar si el tipo de contrato, indefinido o temporal, depende del sexo, se ha realizado un test de corrección de Yates $\left(\chi_{Y}^{2}\right)$, aplicado por un lado a las cooperativas y por otro a las empresas mercantiles para establecer las diferencias entre ambos tipos de organizaciones. Esta prueba -la corrección de Yates para la continuidad- se ha realizado para cada grupo de cotización perteneciente a cada tipo de empresa (cooperativa y mercantil), al objeto de determinar si existen diferencias significativas entre hombres y mujeres en cada uno de ellos. 
Tabla. 2. Clasificación de los grupos de cotización de la MCVL

\begin{tabular}{|l|l|}
\hline Clave & Denominación \\
\hline 1 & Ingenieros, licenciados y alta dirección \\
\hline 2 & Ingenieros técnicos, peritos y ayudantes \\
\hline 3 & Jefes administrativos y de taller \\
\hline 4 & Ayudantes no titulados \\
\hline 5 & Oficiales administrativos \\
\hline 6 & Subalternos \\
\hline 7 & Auxiliares administrativos \\
\hline 8 & Oficiales de primera y de segunda \\
\hline 9 & Oficiales de tercera y especialistas \\
\hline 10 & Mayores de 18 años no cualificados \\
\hline 11 & Trabajadores menores de 18 años \\
\hline
\end{tabular}

Fuente: Guía MCVL

Los grupos de cotización, atendiendo a la MCVL, se dividen en once, siendo el primero de ellos el de ingenieros, licenciados y alta dirección y el último el correspondiente a menores de 18 años (Tabla 2); no obstante, se ha prescindido de este último dado que, para las empresas cooperativas, en este grupo de cotización, no existen trabajadores -según la filtración de datos realizada-.

En lo que respecta a los resultados del test, el sexo no influye en el tipo de contrato para más de la mitad de los grupos de cotización en el caso de las cooperativas, en concreto para los grupos 1 (ingenieros, licenciados y alta dirección), 2 (ingenieros técnicos, peritos y ayudantes), 3 (jefes administrativos y de taller), 5 (oficiales administrativos), 7 (auxiliares administrativos) y 8 (oficiales de primera y de segunda). Por contra, para los cuatro grupos restantes sí existe asociación entre estas dos variables, es decir, el sexo influye en el tipo de contrato, aunque en algunos casos esta significatividad se sitúa en el límite con p-valores por debajo de 0,05 pero muy cercanos a este valor (Tabla 4).

Al analizar la tabla de contingencia, se pone de manifiesto que estas diferencias vienen motivadas sobre todo porque las mujeres superan a los hombres en contratos temporales en los grupos 4, 6 y 9, y en los indefinidos en el grupo de trabajadores mayores de 18 años no cualificados (grupo 10). Ahora bien, cuando esta diferencia viene dada por los contratos temporales, ¿se debe ésta más a que el número de afiliaciones de mujeres es superior y no tanto a un mayor número de personas implicadas? De otra forma, puede ocurrir que exista un comportamiento diferente del número de trabajadores y de afiliaciones, en concreto, que el número de afiliaciones supere al de los hombres, pero no necesariamente porque el colectivo femenino -las mujeres trabajadoras correspondiente a un determinado grupo de cotización- sea mayor, sino que podría deberse a cierta inestabilidad laboral que haga que el número de contratos anuales de las mujeres supere al de los hombres. Para responder a esta cuestión se ha analizado el número de personas implicadas en estas afiliaciones. En este sentido, aunque las afiliaciones medias sean ligeramente superiores para el caso de las mujeres, siendo el grupo de cotización de ayudantes no titulados (grupo 4) el que presenta la diferencia más 
significativa entre ambos colectivos, hay que destacar que efectivamente el número de mujeres supera al de hombres también para el caso de los contratos temporales en estos grupos en los que el test de corrección de Yates ha resultado significativo, es decir, para los que existe asociación entre el sexo y el tipo de contrato (Tabla 5).

Tabla. 3. Tipos de contrato por sexo para cada grupo de cotización

\begin{tabular}{|c|c|c|c|c|c|c|c|}
\hline \multirow{3}{*}{$\begin{array}{l}\text { Grupo de } \\
\text { cotización }\end{array}$} & \multirow{3}{*}{$\begin{array}{l}\text { Tipo de } \\
\text { contrato }\end{array}$} & \multicolumn{3}{|c|}{ COOPERATIVAS } & \multicolumn{3}{|c|}{$\begin{array}{l}\text { EMPRESAS } \\
\text { MERCANTILES }\end{array}$} \\
\hline & & \multicolumn{2}{|l|}{ Sexo } & \multirow{2}{*}{ Total } & \multicolumn{2}{|l|}{ Sexo } & \multirow{2}{*}{ Total } \\
\hline & & Hombre & Mujer & & Hombre & Mujer & \\
\hline \multirow{2}{*}{1} & Indefinidos & 25 & 38 & 63 & 1195 & 666 & 1861 \\
\hline & Temporales & 9 & 25 & 34 & 474 & 409 & 883 \\
\hline \multirow{2}{*}{2} & Indefinidos & 35 & 49 & 84 & 921 & 608 & 1529 \\
\hline & Temporales & 35 & 54 & 89 & 593 & 989 & 1582 \\
\hline \multirow{2}{*}{3} & Indefinidos & 64 & 26 & 90 & 1407 & 579 & 1986 \\
\hline & Temporales & 16 & 6 & 22 & 912 & 478 & 1390 \\
\hline \multirow{2}{*}{4} & Indefinidos & 15 & 21 & 36 & 1002 & 577 & 1579 \\
\hline & Temporales & 11 & 47 & 58 & 2451 & 724 & 3175 \\
\hline \multirow{2}{*}{5} & Indefinidos & 82 & 98 & 180 & 2482 & 3147 & 5629 \\
\hline & Temporales & 35 & 26 & 61 & 1916 & 2269 & 4185 \\
\hline \multirow{2}{*}{6} & Indefinidos & 13 & 14 & 27 & 797 & 556 & 1353 \\
\hline & Temporales & 6 & 28 & 34 & 1789 & 1364 & 3153 \\
\hline \multirow{2}{*}{7} & Indefinidos & 31 & 73 & 104 & 1219 & 3194 & 4413 \\
\hline & Temporales & 60 & 167 & 227 & 3100 & 6300 & 9400 \\
\hline \multirow{2}{*}{8} & Indefinidos & 187 & 44 & 231 & 6139 & 1891 & 8030 \\
\hline & Temporales & 305 & 77 & 382 & 17990 & 5809 & 23799 \\
\hline \multirow{2}{*}{9} & Indefinidos & 97 & 95 & 192 & 2094 & 1611 & 3705 \\
\hline & Temporales & 129 & 350 & 479 & 9084 & 6170 & 15254 \\
\hline \multirow{2}{*}{10} & Indefinidos & 193 & 273 & 466 & 2370 & 3044 & 5414 \\
\hline & Temporales & 1950 & 2096 & 4046 & 26027 & 17578 & 43605 \\
\hline
\end{tabular}

Fuente: Elaboración propia a partir de la MCVL 2015 
Tabla. 4. Test corrección de Yates por sexo y tipo de contrato para cada grupo de cotización

\begin{tabular}{|l|l|l|l|l|l|l|}
\hline & \multicolumn{3}{|l|}{ COOPERATIVAS } & \multicolumn{2}{|l|}{ EMPRESAS MERCANTILES } \\
\hline $\begin{array}{l}\text { Grupo de } \\
\text { cotización }\end{array}$ & $\begin{array}{l}\text { Prueba } \\
\text { test } \\
\text { correción } \\
\text { de Yates }\end{array}$ & $\begin{array}{l}\text { Grados } \\
\text { de } \\
\text { libertad }\end{array}$ & $\begin{array}{l}\text { Sig. } \\
\text { asintótica } \\
(\mathbf{2} \text { caras })\end{array}$ & $\begin{array}{l}\text { Prueba } \\
\text { test } \\
\text { correción } \\
\text { de Yates }\end{array}$ & $\begin{array}{l}\text { Grados } \\
\text { de } \\
\text { libertad }\end{array}$ & $\begin{array}{l}\text { Sig. } \\
\text { asintótica } \\
\text { (2 caras) }\end{array}$ \\
\hline 1 & 1,163 & 1 & 0,281 & 27,438 & 1 & 0,000 \\
\hline 2 & 0,025 & 1 & 0,874 & 160,190 & 1 & 0,000 \\
\hline 3 & 0,000 & 1 & 1,000 & 10,175 & 1 & 0,001 \\
\hline 4 & 4,643 & 1 & 0,031 & 99,453 & 1 & 0,000 \\
\hline 5 & 2,098 & 1 & 0,148 & 2,702 & 1 & 0,100 \\
\hline 6 & 5,184 & 1 & 0,023 & 1,730 & 1 & 0,188 \\
\hline 7 & 0,256 & 1 & 0,613 & 39,835 & 1 & 0,000 \\
\hline 8 & 0,053 & 1 & 0,818 & 2,371 & 1 & 0,124 \\
\hline 9 & 33,098 & 1 & 0,000 & 11,210 & 1 & 0,001 \\
\hline 10 & 7,432 & 1 & 0,006 & 499,732 & 1 & 0,000 \\
\hline
\end{tabular}

Fuente: Elaboración propia a partir de la MCVL 2015

Tabla. 5. Afiliaciones medias en cooperativas para los grupos de cotización cuatro, seis, nueve y diez

\begin{tabular}{|l|l|lr|l|l|}
\hline $\begin{array}{l}\text { Grupo de } \\
\text { cotización }\end{array}$ & Sexo & $\begin{array}{l}\text { Número de } \\
\text { afiliaciones } \\
\text { contratos temporales }\end{array}$ & $\begin{array}{l}\text { Personas } \\
\text { implicadas }\end{array}$ & $\begin{array}{l}\text { Afiliaciones } \\
\text { me dias por } \\
\text { persona }\end{array}$ \\
\hline \multirow{2}{*}{4} & Hombres & 11 & 9 & 1,22 \\
\cline { 2 - 6 } & Mujeres & 47 & 17 & 2,76 \\
\hline \multirow{2}{*}{6} & Hombres & 6 & 6 & 1 \\
\hline \multirow{2}{*}{9} & Mujeres & 28 & 17 & 1,65 \\
\hline \multirow{2}{*}{10} & Hombres & 129 & 76 & 1,70 \\
\cline { 2 - 4 } & 350 & 181 & 1,93 \\
\hline & Mujbres & 1950 & 1202 & 1,62 \\
\cline { 2 - 5 } & Mujeres & 2096 & 1388 & 1,51 \\
\hline
\end{tabular}

Fuente: Elaboración propia a partir de la MCVL 2015

En lo que respecta a la jornada de trabajo, mediante la variable coeficiente de tiempo parcial, se ha identificado asimismo si este mayor número de mujeres con contratos temporales podría deberse a que son a tiempo parcial. En primer lugar se ha aplicado, también en este caso, el test de corrección de Yates por sexo y jornada laboral para cada tipo de contrato, para determinar previamente si la distribución es o no homogénea. El resultado es que existe asociación entre la variable sexo y el tipo de jornada para cada tipo de contrato ( $p$-valores inferiores a 0,05 ), es decir, el sexo influye en que la jornada laboral sea completa o parcial tanto para los indefinidos como temporales (Tabla 7). Esta significatividad del test de Yates 
puede explicarse porque el porcentaje de mujeres con jornada parcial es superior tanto para el caso de los contratos indefinidos como el de los temporales (Tabla 6).

Para el caso de las empresas mercantiles, los resultados son significativamente diferentes a los de las cooperativas: la relación entre las variables sexo y tipo de contrato se producen en un mayor número de grupos de cotización que en el caso de las cooperativas. No existe asociación únicamente para los grupos de cotización de oficiales administrativos, subalternos y oficiales de primera y segunda (Tabla 4).

Tabla. 6. Porcentaje de trabajadores por jornada laboral, tipo de contrato y sexo

\begin{tabular}{|c|c|c|c|c|c|c|c|}
\hline \multirow{3}{*}{$\begin{array}{lr}\text { TIPO DE } \\
\text { CONTRATO }\end{array}$} & \multirow{3}{*}{$\begin{array}{l}\text { JORNADA } \\
\text { LABORAL }\end{array}$} & \multicolumn{3}{|c|}{ COOPERATIVAS } & \multicolumn{3}{|c|}{$\begin{array}{l}\text { EMPRESAS } \\
\text { MERCANTILES }\end{array}$} \\
\hline & & \multicolumn{2}{|l|}{ Sexo } & \multirow{2}{*}{ Total } & \multicolumn{2}{|l|}{ Sexo } & \multirow{2}{*}{ Total } \\
\hline & & Hombre & Mujer & & Hombre & Mujer & \\
\hline \multirow{3}{*}{ Indefinido } & Completa & $95 \%$ & $72,9 \%$ & $84,2 \%$ & $88,8 \%$ & $54,1 \%$ & $74,2 \%$ \\
\hline & Parcial & $5 \%$ & $27,1 \%$ & $15,8 \%$ & $11,2 \%$ & $45,9 \%$ & $25,8 \%$ \\
\hline & Total & $100 \%$ & $100 \%$ & $100 \%$ & $100 \%$ & $100 \%$ & $100 \%$ \\
\hline \multirow{3}{*}{ Temporal } & Completa & $92,1 \%$ & $86,3 \%$ & $89,1 \%$ & $72,1 \%$ & $48,2 \%$ & $62,3 \%$ \\
\hline & Parcial & $7,9 \%$ & $13,7 \%$ & $10,9 \%$ & $27,9 \%$ & $51,8 \%$ & $37,7 \%$ \\
\hline & Total & $100 \%$ & $100 \%$ & $100 \%$ & $100 \%$ & $100 \%$ & $100 \%$ \\
\hline \multirow{3}{*}{ Total } & Completa & $92,9 \%$ & $83,1 \%$ & $87,8 \%$ & $79 \%$ & $50,7 \%$ & $67,3 \%$ \\
\hline & Parcial & $7,1 \%$ & $16,9 \%$ & $12,2 \%$ & $21 \%$ & $49,3 \%$ & $32,7 \%$ \\
\hline & Total & $100 \%$ & $100 \%$ & $100 \%$ & $100 \%$ & $100 \%$ & $100 \%$ \\
\hline
\end{tabular}

Fuente: Elaboración propia a partir de la MCVL 2015 
Tabla. 7. Test corrección de Yates por sexo y tipo de jornada para cada tipo de contrato

\begin{tabular}{|l|l|l|l|l|l|l|}
\hline & \multicolumn{2}{|l|}{ COOPERATIVAS } & \multicolumn{2}{l|}{ EMPRESAS MERCANTILES } \\
\hline $\begin{array}{l}\text { Tipo de } \\
\text { contrato }\end{array}$ & $\begin{array}{l}\text { Prueba } \\
\text { test } \\
\text { correción } \\
\text { de Yates }\end{array}$ & $\begin{array}{l}\text { Grados } \\
\text { de } \\
\text { libertad }\end{array}$ & $\begin{array}{l}\text { Sig. } \\
\text { asintótica } \\
\text { (2 caras) }\end{array}$ & $\begin{array}{l}\text { Prueba } \\
\text { test } \\
\text { correción } \\
\text { de Yates }\end{array}$ & $\begin{array}{l}\text { Grados } \\
\text { de } \\
\text { libertad }\end{array}$ & $\begin{array}{l}\text { Sig. } \\
\text { asintótica } \\
\text { (2 caras) }\end{array}$ \\
\hline Indefinido & 98,099 & 1 & 0,000 & 4274,169 & 1 & 0,000 \\
\hline Temporal & 27,418 & 1 & 0,000 & 2246,855 & 1 & 0,000 \\
\hline Total & 95,379 & 1 & 0,000 & 5852,674 & 1 & 0,000 \\
\hline
\end{tabular}

Fuente: Elaboración propia a partir de la MCVL 2015

A diferencia de lo que ocurre en las sociedades cooperativas, esta influencia del sexo sobre el tipo de contrato se debe a que en la mayoría de los grupos de cotización los hombres superan a las mujeres tanto en los indefinidos como en los temporales. Únicamente en el grupo de cotización 7, el de auxiliares administrativos, los contratos a mujeres son superiores al de hombres en ambas modalidades (Tabla 3).

En lo que respecta a la jornada laboral, al igual que para el caso de las cooperativas, el sexo influye en ésta para cada tipo de contrato (los test de corrección de Yates han resultado significativos en cada uno de ellos a un nivel de confianza del 99\%) (Tabla 7). Aunque también existe un mayor número de mujeres a tiempo parcial que de hombres, esta proporción es muy superior que para el caso de las cooperativas: en general, el 49,3\% de las mujeres que trabajan en empresas mercantiles lo hacen a jornada parcial frente a un $21 \%$ para el caso de los hombres, mientras que en las cooperativas esta cifra es del 16,9\% y 7,1\% respectivamente (Tabla 6). En la actualidad, la situación de conciliación entre la vida familiar y la laboral todavía está dirigida principalmente a mujeres, lo que demuestra cierto desequilibrio frente a los hombres en la igualdad de oportunidades.

Sin distinguir por sexo, hay que señalar que la proporción de contratos a jornada completa es mucho más elevada para el caso de las sociedades cooperativas $(87,8 \%)$ que en las empresas mercantiles $(67,3 \%)$.

Uno de los resultados más interesantes, que se derivan del estudio, es que para los grupos de cotización más elevados en sociedades cooperativas, que requieren de ciertas capacidades directivas, de liderazgo y formación, el sexo no influye en el tipo de contrato, no ocurriendo lo mismo en las empresas mercantiles en las que se observa un mayor número de hombres que de mujeres con cierta estabilidad laboral (contratos indefinidos). Estas sociedades convencionales, tradicionalmente, han contado con una proporción de empleo femenino bajo en los estratos laborales superiores, relacionados con la toma de decisiones organizativas y de dirección; en la ES, por su propia configuración y principios y valores, se refleja mejor la implicación y el compromiso con la igualdad de oportunidades desde la perspectiva de género, de ahí que la presencia de la mujer en estos puestos de trabajo, que 
requieren capacidades directivas y de formación, sea más significativa que en las empresas convencionales.

Por otro lado, aunque el tipo de jornada laboral no es determinante para identificar la mayor calidad del empleo en las sociedades cooperativas, ya que se requeriría información acerca de si ésta ha sido por voluntad propia o no, hay que destacar la mayor proporción de trabajadores con contratos a tiempo completo en las cooperativas que en las empresas mercantiles, hecho que puede interpretarse en la línea de una mayor calidad del empleo en estas organizaciones de ES.

Por sexo, dentro del grupo de trabajadores en cooperativas, la proporción de mujeres con contrato a tiempo parcial es superior que la de hombres; sin embargo, en comparación con las empresas mercantiles, esta proporción es menor. Estos resultados coinciden con los de Martínez, Arcas y García (2011) y Santero y Castro (2016) para el caso español, quienes afirman que de esta forma las mujeres cuentan con mayores oportunidades en las empresas de ES si demandan puestos de trabajo a jornada completa respecto a las empresas mercantiles o convencionales.

\subsection{Empleo por grandes grupos de edad}

Según los datos que figuran en la MCVL, el mayor grueso de las afiliaciones en Andalucía corresponde a personas con edades comprendidas entre 30 y 45 años, tanto para las cooperativas como para las empresas mercantiles con el $45 \%$ y $48 \%$ del total de las afiliaciones respectivamente. Estos datos coinciden con el número de personas implicadas en estas organizaciones, es decir, el 45\% de las afiliaciones pertenece a trabajadores entre 30 y 45 años, representando éstos también un $45 \%$ sobre el total de empleados, sucediendo lo mismo para las empresas convencionales que representan el 48\% (Tabla 8).

Tabla. 8. Porcentaje de afiliaciones y personas por edades

\begin{tabular}{|l|l|l|l|l|}
\hline \multirow{2}{*}{ EDAD } & \multicolumn{3}{|l|}{ COOPERATIVAS } & \multicolumn{2}{l|}{ EMPRESAS MERCANTILES } \\
\cline { 2 - 5 } & Afiliaciones & Personas & Afiliaciones & Personas \\
\hline Menos de 30 años & $23 \%$ & $24 \%$ & $27 \%$ & $23 \%$ \\
\hline Entre 30 y 45 & $45 \%$ & $45 \%$ & $48 \%$ & $48 \%$ \\
\hline Más de 45 & $32 \%$ & $31 \%$ & $25 \%$ & $29 \%$ \\
\hline
\end{tabular}

Fuente: Elaboración propia a partir de la MCVL 2015

Para el caso de los trabajadores mayores de 45 años, este porcentaje es superior, tanto para las afiliaciones como para el número de personas empleadas sobre el total en las sociedades cooperativas: éstos representan el $32 \%$ de las afiliaciones y el $31 \%$ de los trabajadores, mientras que en las empresas mercantiles este colectivo aglutina al 25\% el 29\% respectivamente. Estos resultados pueden estar indicando la lucha contra la exclusión social y el fomento de la inserción laboral que promueven las organizaciones de ES, en este caso, para el colectivo de mayores de 45 años, 
con una problemática importante de acceso al mercado laboral; por otro lado, es otro indicador de la estabilidad en el empleo.

Para trabajadores menores de 30 años, los datos ponen de manifiesto que, a nivel de afiliaciones, éstos son mayores en las empresas mercantiles que en las cooperativas, pero que, sin embargo, en lo que se refiere a personas empleadas, el porcentaje es similar. Esto significa que existe una mayor estabilidad en el empleo para los jóvenes en estas organizaciones de ES.

Gráfico. 1. Afiliaciones medias por grupos de edad

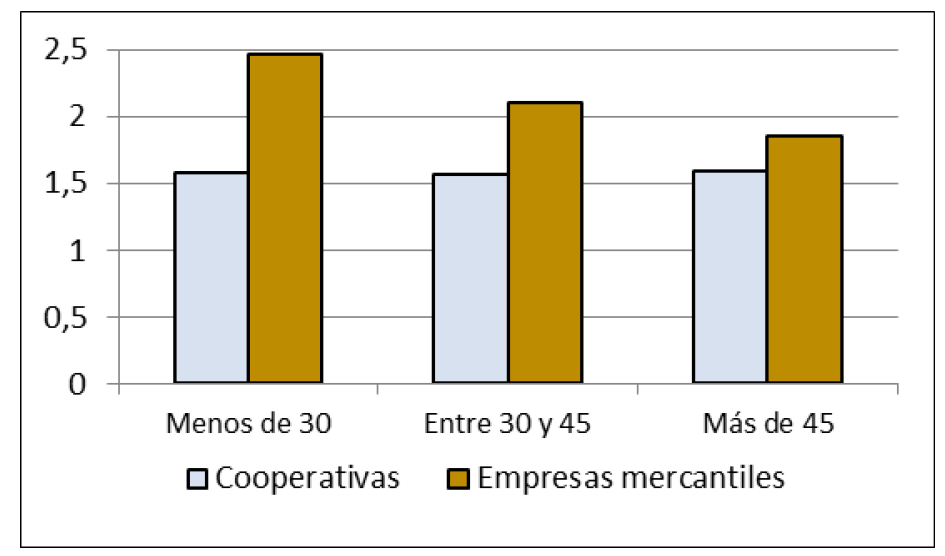

Fuente: Elaboración propia a partir de la MCVL 2015

Anteriormente se ha señalado que la cifra de afiliaciones anuales medias por persona es inferior para el caso de las empresas cooperativas, lo que ocurre también al analizarlas por grupos de edad. La diferencia más significativa se produce con los menores de 30 años ya que cada trabajador de una empresa mercantil ha tenido una media de 2,46 afiliaciones en el año, mientras que en las cooperativas la cifra ha sido de 1,58; es decir, entre los más jóvenes, existe una mayor temporalidad y precariedad en el empleo en las sociedades convencionales que en las cooperativas (Gráfico 1). Que las proporciones de trabajadores jóvenes por un lado -en concreto los menores de 30 años-, y los mayores de 45, por otro, sea superior en las empresas cooperativas que en el sector tradicional mercantil, supone un valor social añadido. La última crisis iniciada a finales de 2007, comienzos de 2008, ha dificultado la (re) incorporación al mercado laboral de estos colectivos sociales más vulnerables y en mayor riesgo de exclusión social.

Sin embargo, al identificar las diferencias por tipo de contrato -indefinido o temporal-, la proporción de afiliaciones temporales en las cooperativas supera en todos los grupos de edad a la de las empresas mercantiles (Tabla 9). Comparando esto con el resultado anterior, el valor inferior de las afiliaciones medias anuales por trabajador en las cooperativas, lleva a plantear la idea de que los contratos en éstas tengan una duración superior que en las empresas convencionales. Es decir, a pesar de una mayor temporalidad en las cooperativas, éstas presentan un número 
medio de afiliaciones inferior que las sociedades mercantiles, lo que se traduce en mayor estabilidad de estos contratos temporales, con una duración superior.

\subsection{Empleo según secciones de actividad}

El objeto de identificar el diferente peso de las actividades económicas en términos de empleo cobra interés ya que ofrece una aproximación al tipo de cooperativismo que existe en Andalucía en términos comparados con el sector empresarial tradicional.

Hay que destacar que gran parte de la temporalidad existente en el empleo generado por las sociedades cooperativas es debida a la estacionalidad del sector primario. Este sector en Andalucía representa el 47\% del total de las afiliaciones en estas organizaciones y solo las temporales el 45\%. Esta temporalidad se manifiesta aún más cuando se identifican el número de trabajadores para estas afiliaciones: tan solo el 4\% de los trabajadores del sector son indefinidos siendo la proporción total de trabajadores en cooperativas del sector primario el 42\% (Tabla 9).

Para el caso de las empresas mercantiles, la temporalidad en el sector primario también está presente de manera significativa, representando los indefinidos sobre el total de trabajadores del sector tan solo el $0,7 \%$. No obstante, a diferencia de las sociedades cooperativas, los trabajadores en empresas mercantiles del sector agrario y ganadero tan tolo son el 11,1\% (Tabla 9).

Tabla. 9. Porcentaje de afiliaciones y trabajadores por secciones de actividad

\begin{tabular}{|c|c|c|c|c|c|c|c|c|c|}
\hline \multirow{3}{*}{$\begin{array}{l}\text { Sección de actividad } \\
\text { (CNAE 2009) }\end{array}$} & \multirow{3}{*}{$\begin{array}{l}\text { Tipo de } \\
\text { contrato }\end{array}$} & \multicolumn{4}{|c|}{ Afiliaciones } & \multicolumn{4}{|c|}{ Personas } \\
\hline & & \multicolumn{2}{|l|}{ Coop. } & \multicolumn{2}{|c|}{$\begin{array}{l}\text { Emp. } \\
\text { mercantiles }\end{array}$} & \multicolumn{2}{|l|}{ Coop. } & \multicolumn{2}{|c|}{$\begin{array}{l}\text { Emp. } \\
\text { mercantiles }\end{array}$} \\
\hline & & $\%$ & $\% /$ total & $\%$ & $\% /$ total & $\%$ & $\% /$ total & $\%$ & $\% /$ total \\
\hline \multirow{3}{*}{$\begin{array}{l}\text { A. Agricultura, } \\
\text { ganadería, silvicultura } \\
\text { y pesca }\end{array}$} & Inde finidos & 3,9 & 1,9 & 4,2 & 0,5 & 4,0 & 1,7 & & 0,7 \\
\hline & Temporales & 96,1 & 45,4 & 95,8 & 11,1 & 96,0 & 40,5 & 94,0 & 10,5 \\
\hline & Total & 100,0 & 47,3 & 100,0 & 11,6 & 100,0 & 42,2 & 100,0 & 11,1 \\
\hline \multirow{3}{*}{$\begin{array}{ll}\text { B. } & \text { Industrias } \\
\text { extractivas } & \end{array}$} & Inde finidos & 0,0 & 0,0 & 56,2 & 0,1 & 0,0 & 0,0 & 63,4 & 0,1 \\
\hline & Temporales & 100,0 & 0,0 & 43,8 & 0,1 & 100,0 & 0,0 & 36,6 & 0,1 \\
\hline & Total & 100,0 & 0,0 & 100,0 & 0,1 & 100,0 & 0,0 & 100,0 & 0,2 \\
\hline \multirow{3}{*}{$\begin{array}{l}\text { C. Industria } \\
\text { manufacturera }\end{array}$} & Inde finidos & 53,9 & 5,1 & 73,2 & 3,2 & 54,6 & 4,6 & 55,2 & 5,1 \\
\hline & Temporales & 46,1 & 4,3 & 57,7 & 4,4 & 45,4 & 3,8 & 44,8 & 4,1 \\
\hline & Total & 100,0 & 9,4 & 100,0 & 7,6 & 100,0 & 8,4 & 100,0 & 9,2 \\
\hline \multirow{3}{*}{$\begin{array}{l}\text { D. Suministro de } \\
\text { energía eléctrica, gas, } \\
\text { vapor y aire } \\
\text { acondicionado }\end{array}$} & Inde finidos & 100,0 & 0,0 & 82,3 & 0,1 & 100,0 & 0,0 & 84,5 & 0,2 \\
\hline & Temporales & 0,0 & 0,0 & 17,7 & 0,0 & 0,0 & 0,0 & 15,5 & 0,0 \\
\hline & Total & 100,0 & 0,0 & 100,0 & 0,2 & 100,0 & 0,0 & 100,0 & 0,3 \\
\hline \multirow{2}{*}{$\begin{array}{l}\text { E. Suministro de agua, } \\
\text { actividades de }\end{array}$} & Inde finidos & 100,0 & 0,0 & 43,5 & 0,5 & 100,0 & 0,0 & 65,8 & 0,8 \\
\hline & Temporales & 0,0 & 0,0 & 56,5 & 0,6 & 0,0 & 0,0 & 34,2 & 0,4 \\
\hline
\end{tabular}




\begin{tabular}{|c|c|c|c|c|c|c|c|c|c|}
\hline \multirow{4}{*}{$\begin{array}{l}\text { Sección de actividad } \\
\text { (CNAE 2009) } \\
\text { saneamiento, gestión } \\
\text { de residuos y } \\
\text { descontaminación }\end{array}$} & \multirow{3}{*}{$\begin{array}{l}\text { Tipo de } \\
\text { contrato }\end{array}$} & \multicolumn{4}{|c|}{ Afiliaciones } & \multicolumn{4}{|c|}{ Personas } \\
\hline & & \multicolumn{2}{|l|}{ Coop. } & \multicolumn{2}{|c|}{$\begin{array}{l}\text { Emp. } \\
\text { me rcantiles }\end{array}$} & \multicolumn{2}{|l|}{ Coop. } & \multicolumn{2}{|c|}{$\begin{array}{l}\text { Emp. } \\
\text { mercantiles }\end{array}$} \\
\hline & & $\%$ & $\% /$ total & $\%$ & $\% /$ total & $\%$ & $\% /$ total & $\%$ & $\% /$ total \\
\hline & Total & 100,0 & 0,0 & 100,0 & 1,1 & 100,0 & 0,0 & 100,0 & 1,2 \\
\hline \multirow{3}{*}{ F. Construcción } & Inde finidos & 7,8 & 0,1 & 13,6 & 1,1 & 13,2 & 0,2 & 22,5 & 1,8 \\
\hline & Temporales & 92,2 & 1,7 & 86,4 & 7,3 & 86,8 & 1,3 & 77,5 & 6,2 \\
\hline & Total & 100,0 & 1,9 & 100,0 & 8,4 & 100,0 & 1,5 & 100,0 & 8,0 \\
\hline \multirow{3}{*}{$\begin{array}{l}\text { G. Comercio al por } \\
\text { mayor y al por menor; } \\
\text { reparación de } \\
\text { vehículos de motor y } \\
\text { motocicletas }\end{array}$} & Inde finidos & 34,9 & 7,6 & 48,4 & 6,1 & 35,5 & 10,8 & 23,9 & 5,5 \\
\hline & Temporales & 65,1 & 14,2 & 51,6 & 6,5 & 64,5 & 19,7 & 76,1 & 17,4 \\
\hline & Total & 100,0 & 21,8 & 100,0 & 12,6 & 100,0 & 30,5 & 100,0 & 22,8 \\
\hline \multirow{3}{*}{$\begin{array}{l}\text { H. Transporte y } \\
\text { almacenamiento }\end{array}$} & Inde finidos & 32,9 & 0,4 & 28,1 & 1,4 & 40,0 & 0,5 & 47,8 & 2,2 \\
\hline & Temporales & 67,1 & 0,8 & 71,9 & 3,6 & 60,0 & 0,8 & 52,2 & 2,4 \\
\hline & Total & 100,0 & 1,2 & 100,0 & 4,9 & 100,0 & 1,3 & 100,0 & 4,6 \\
\hline \multirow{3}{*}{ I. Hostelería } & Inde finidos & 9,6 & 0,2 & 18,6 & 3,4 & 20,3 & 0,3 & 33,7 & 3,8 \\
\hline & Temporales & 90,4 & 1,9 & 81,4 & 14,8 & 79,7 & 1,0 & 66,3 & 7,5 \\
\hline & Total & 100,0 & 2,1 & 100,0 & 18,2 & 100,0 & 1,3 & 100,0 & 11,3 \\
\hline \multirow{3}{*}{$\begin{array}{l}\text { J. Información y } \\
\text { comunicaciones }\end{array}$} & Inde finidos & 30,0 & 0,1 & 42,7 & 0,7 & 60,0 & 0,1 & 54,5 & 1,2 \\
\hline & Temporales & 70,0 & 0,2 & 57,3 & 0,9 & 40,0 & 0,1 & 45,5 & 1,0 \\
\hline & Total & 100,0 & 0,3 & 100,0 & 1,7 & 100,0 & 0,2 & 100,0 & 2,2 \\
\hline \multirow{3}{*}{$\begin{array}{l}\text { K. Actividades } \\
\text { financieras } y \text { de } \\
\text { seguros }\end{array}$} & Inde finidos & 87,3 & 2,2 & 86,1 & 0,9 & 91,9 & 3,0 & 89,3 & 1,7 \\
\hline & Temporales & 12,7 & 0,3 & 13,9 & 0,2 & 8,1 & 0,3 & 10,7 & 0,2 \\
\hline & Total & 100,0 & 2,5 & 100,0 & 1,1 & 100,0 & 3,2 & 100,0 & 1,9 \\
\hline \multirow{3}{*}{$\begin{array}{l}\text { L. Actividades } \\
\text { inmobiliarias }\end{array}$} & Indefinidos & 0,0 & 0,0 & 52,0 & 0,2 & 0,0 & 0,0 & 56,0 & 0,4 \\
\hline & Temporales & 0,0 & 0,0 & 48,0 & 0,2 & 0,0 & 0,0 & 44,0 & 0,3 \\
\hline & Total & 0,0 & 0,0 & 100,0 & 0,4 & 0,0 & 0,0 & 100,0 & 0,6 \\
\hline \multirow{3}{*}{$\begin{array}{l}\text { M. Actividades } \\
\text { profesionales, } \\
\text { científicas y técnicas }\end{array}$} & Indefinidos & 32,3 & 0,1 & 37,3 & 1,1 & 41,7 & 0,2 & 50,4 & 1,8 \\
\hline & Temporales & 67,7 & 0,3 & 62,7 & 1,9 & 58,3 & 0,3 & 49,6 & 1,8 \\
\hline & Total & 100,0 & 0,4 & 100,0 & 3,0 & 100,0 & 0,5 & 100,0 & 3,6 \\
\hline \multirow{3}{*}{$\begin{array}{l}\text { N. Actividades } \\
\text { administrativas y } \\
\text { servicios auxiliares }\end{array}$} & Indefinidos & 14,4 & 0,4 & 15,4 & 2,9 & 25,2 & 0,5 & 29,9 & 3,8 \\
\hline & Temporales & 85,6 & 2,2 & 84,6 & 15,7 & 74,8 & 1,6 & 70,1 & 8,9 \\
\hline & Total & 100,0 & 2,6 & 100,0 & 18,5 & 100,0 & 2,1 & 100,0 & 12,7 \\
\hline \multirow{3}{*}{$\begin{array}{lr}\text { O. Administración } \\
\text { pública y defensa; } \\
\text { seguridad } \\
\text { obligatoria }\end{array}$} & Inde finidos & 0,0 & 0,0 & 24,6 & 0,0 & 0,0 & 0,0 & 34,0 & 0,0 \\
\hline & Temporales & 0,0 & 0,0 & 75,4 & 0,0 & 0,0 & 0,0 & 66,0 & 0,0 \\
\hline & Total & 0,0 & 0,0 & 100,0 & 0,0 & 0,0 & 0,0 & 100,0 & 0,1 \\
\hline P. Educación & Inde finidos & 30,2 & 1,2 & 28,0 & 0,7 & 39,7 & 1,3 & 32,4 & 0,8 \\
\hline
\end{tabular}




\begin{tabular}{|c|c|c|c|c|c|c|c|c|c|}
\hline \multirow{5}{*}{$\begin{array}{l}\text { Sección de actividad } \\
\text { (CNAE 2009) }\end{array}$} & \multirow{4}{*}{$\begin{array}{l}\text { Tipo de } \\
\text { contrato }\end{array}$} & \multicolumn{4}{|c|}{ Afiliaciones } & \multicolumn{4}{|c|}{ Personas } \\
\hline & & \multicolumn{2}{|l|}{ Coop. } & \multicolumn{2}{|c|}{$\begin{array}{l}\text { Emp. } \\
\text { me rcantiles }\end{array}$} & \multicolumn{2}{|l|}{ Coop. } & \multicolumn{2}{|c|}{$\begin{array}{l}\text { Emp. } \\
\text { me rcantiles }\end{array}$} \\
\hline & & $\%$ & $\% /$ total & $\%$ & $\% /$ total & $\%$ & $\% /$ total & $\%$ & $\% /$ total \\
\hline & & 69,8 & 2,8 & 72,0 & 1,8 & 41,0 & 1,3 & 67,6 & 1,7 \\
\hline & Total & 100,0 & 4,0 & 100,0 & 2,5 & 100,0 & 3,2 & 100,0 & 2,6 \\
\hline \multirow{3}{*}{$\begin{array}{l}\text { Q. Actividades } \\
\text { sanitarias y de } \\
\text { servicios sociales }\end{array}$} & Indefinidos & 33,2 & 1,9 & 32,1 & 1,3 & 52,6 & 2,5 & 50,5 & 2,0 \\
\hline & Temporales & 66,8 & 3,7 & 67,9 & 2,7 & 47,4 & 2,2 & 49,5 & 1,9 \\
\hline & Total & 100,0 & 5,6 & 100,0 & 3,9 & 100,0 & 4,7 & 100,0 & 3,9 \\
\hline \multirow{3}{*}{$\begin{array}{l}\mathrm{R} . \quad \text { Actividades } \\
\text { artísticas, recreativas y } \\
\text { de entretenimiento }\end{array}$} & Inde finidos & 18,9 & 0,1 & 13,1 & 0,4 & 27,3 & 0,1 & 22,2 & 0,5 \\
\hline & Temporales & 81,1 & 0,4 & 86,9 & 2,8 & 72,7 & 0,3 & 77,8 & 1,9 \\
\hline & Total & 100,0 & 0,5 & 100,0 & 3,2 & 100,0 & 0,4 & 100,0 & 2,4 \\
\hline \multirow{3}{*}{ S. Otros servicios } & Inde finidos & 22,7 & 0,1 & 42,9 & 0,4 & 25,0 & 0,1 & 47,0 & 0,6 \\
\hline & Temporales & 77,3 & 0,2 & 57,1 & 0,5 & 75,0 & 0,2 & 53,0 & 0,6 \\
\hline & Total & 100,0 & 0,3 & 100,0 & 0,9 & 100,0 & 0,3 & 100,0 & 1,2 \\
\hline \multirow{2}{*}{$\begin{array}{l}\text { T. Actividades de los } \\
\text { hogares } \\
\text { empleadores como } \\
\text { personal doméstico; } \\
\text { actividades de los } \\
\text { hogares } \\
\text { productores de bienes } \\
\text { y servicios para uso } \\
\text { propio }\end{array}$} & $\begin{array}{l}\text { Indefinidos } \\
\text { Temporales }\end{array}$ & $\begin{array}{l}0 \\
0\end{array}$ & $\begin{array}{l}0 \\
0\end{array}$ & $\begin{array}{l}35,7 \\
64,3\end{array}$ & $\begin{array}{l}0,0 \\
0,0\end{array}$ & $\begin{array}{l}0,0 \\
0,0\end{array}$ & $\begin{array}{l}0,0 \\
0,0\end{array}$ & $\begin{array}{l}37,0 \\
63,0\end{array}$ & $\begin{array}{l}0,0 \\
0,0\end{array}$ \\
\hline & Total & 0 & 0 & 100,0 & 0,0 & 0,0 & 0,0 & 100,0 & 0,0 \\
\hline
\end{tabular}

Fuente: Elaboración propia a partir de la MCVL 2015

Si se comparan los resultados de los trabajadores implicados en cooperativas con contratos temporales, éstos son el $73 \%$, mientras que para el caso de las empresas mercantiles la cifra es del $67 \%$, lo que se debe, en gran medida, a la situación del sector primario descrita.

Las actividades de las cooperativas que mayor empleo generan, atendiendo a las afiliaciones por orden decreciente, son la agricultura, ganadería silvicultura y pesca (47,3\%), comercio al por mayor y al por menor; reparación de vehículos de motor y motocicletas $(21,8 \%)$, industria manufacturera $(9,4 \%)$, aglutinando al $79 \%$ del total de afiliaciones en estas organizaciones y al $81 \%$ de trabajadores directos implicados. Para las empresas mercantiles, el orden es, para el caso de las afiliaciones, actividades administrativas y de servicios auxiliares (18,5\%), hostelería (18,2\%) y comercio al por mayor y al por menor; reparación de vehículos de motor y motocicletas con el 12,7\%, 11,3\% y 22,8\% de los trabajadores implicados respectivamente.

Comparando los resultados para ambos tipos de organizaciones, las sociedades cooperativas tienen una proporción de trabajadores -en relación al total- superior al de las empresas mercantiles en las actividades de agricultura, ganadería, 
silvicultura y pesca; comercio al por mayor y al por menor y reparación de vehiculos de motor y motocicletas; actividades financieras y de seguros; educación y actividades sanitarias y de servicios sociales. Es decir, generan una proporción relativa de empleo superior al caso de las empresas mercantiles, lo que indica que el cooperativismo se localiza en estos sectores en mayor medida que el sector empresarial tradicional. Las empresas mercantiles destacan significativamente respecto a las cooperativas en las actividades de construcción, hostelería, actividades profesionales, cientificas y técnicas y actividades administrativas y servicios auxiliares, entre otras (Tabla 9).

Tradicionalmente ha existido una fuerte presencia del cooperativismo en el sector primario andaluz, lo que aquí se ha evidenciado al analizarlo en materia de empleo. No obstante, a pesar de su importancia cuantitativa, se trata de un empleo inestable, con una alta tasa de temporalidad, que puede responder tanto al carácter estacional del propio sector como a determinado grado de eventualidad, siendo esto un problema generalizado de carácter estructural de la comunidad autónoma de Andalucía. Por otro lado, el cooperativismo no logra consolidarse en las actividades profesionales, científicas y técnicas y las administrativas y de servicios auxiliares, estando el sector empresarial tradicional muy por encima en cuanto a la proporción de empleo generado en las citadas actividades. A partir de esto, se ha hecho necesario identificar si estas diferencias, entre ambos tipos de organización, tienen lugar también desde el ámbito formativo, desde la cualificación de los trabajadores.

\subsection{Nivel educativo por secciones de actividad}

Uno de los principios de las sociedades cooperativas es que fomentan la educación, formación e información de sus miembros, con el fin de que puedan contribuir, aprovechando este potencial, al desarrollo de sus organizaciones (principio quinto). En esta línea, en el presente apartado, se identifica la cualificación de estos trabajadores, poniéndose en evidencia así el posible cumplimiento de este principio.

Para obtener una aproximación a la cualificación de los trabajadores, se ha utilizado como variable proxy el nivel educativo que figura para cada uno de ellos en la MCVL. En concreto, se ha identificado, mediante un análisis descriptivo, el nivel educativo tanto de las empresas cooperativas como de las mercantiles por secciones de actividad.

A partir de la clasificación de los niveles educativos que figura en la MCVL (Tabla 10), se ha establecido la siguiente tipología en orden creciente:

1. Sin titulación o nivel inferior a graduado escolar (códigos 10 y 12).

2. Graduado escolar o equivalente (códigos 20, 21 y 22).

3. Bachiller, formación profesional u otras titulaciones medias (códigos del 40 al 43).

4. Estudios universitarios y de postgrado (códigos del 44 al 48). 
Tabla. 10. Niveles educativos de la MCVL

\begin{tabular}{|l|l|}
\hline Código & Descripción del nivel educativo \\
\hline 00 & No aplicable por ser menor de 16 años \\
\hline 10 & NO SABE LEER NI ESCRIBIR \\
\hline 11 & No sabe leer ni escribir \\
\hline 20 & TITULACIÓN INFERIOR A GRADUADO ESCOLAR \\
\hline 21 & Sin Estudios \\
\hline 22 & $\begin{array}{l}\text { Enseñanza Primaria incompleta. Cinco cursos de EGB o equivalente o } \\
\text { Certificado de Escolaridad o equivalente }\end{array}$ \\
\hline 30 & GRADUADO ESCOLAR O EQUIVALENTE \\
\hline 31 & Bachiller Elemental. Graduado Escolar. EGB completa. Primaria completa. ESO \\
\hline 32 & $\begin{array}{l}\text { Formación Profesional de Primer Grado. Formación profesional de Grado Medio. } \\
\text { Oficialía Industrial }\end{array}$ \\
\hline 40 & $\begin{array}{l}\text { BACHILLER, FORMACIÓN PROFESIONAL DE SEGUNDO GRADO O } \\
\text { TITULOS EQUIVALENTES O SUPERIORES }\end{array}$ \\
\hline 41 & $\begin{array}{l}\text { Formación Profesional de Segundo Grado. Formación profesional de Grado } \\
\text { Superior. Maestría Industrial }\end{array}$ \\
\hline 42 & Bachiller Superior. BUP. Bachiller LOGSE \\
\hline 43 & $\begin{array}{l}\text { Otras titulaciones medias (Auxiliar de Clínica, Secretariado, Programador de } \\
\text { informática. Auxiliar de vuelo. Diplomados en Artes y Oficios, etc..) }\end{array}$ \\
\hline 44 & $\begin{array}{l}\text { Diplomados en Escuelas Universitarias (Empresariales, Profesorado de EGB, } \\
\text { ATS y similares) }\end{array}$ \\
\hline 45 & Arquitecto o Ingeniero Técnico \\
\hline 46 & Licenciado Universitario. Arquitecto o Ingeniero Superior. Grado universitario \\
\hline 47 & Titulados de Estudios Superiores no Universitarios \\
\hline 48 & $\begin{array}{l}\text { Doctorado y Estudios de postgrado o especialización para Licenciados. Máster } \\
\text { Desconocido }\end{array}$ \\
\hline
\end{tabular}

Fuente: Guía MCVL 2015

No se han considerado las afiliaciones de la muestra a las que no ha sido de aplicación el nivel educativo por ser menor de 16 años (código 00) ni aquéllas para las que el dato es desconocido (código 99).

Una vez realizada esta clasificación, se ha desarrollado un test chi-cuadrado de Pearson para determinar si el nivel educativo depende de que el tipo de empresa sea cooperativa o mercantil para cada sección de actividad. No obstante, a diferencia del apartado anterior, determinadas secciones han sido agrupadas por razones metodológicas ya que para algunos casos la frecuencia observada ha sido menor que cinco. 
Tabla. 11. Niveles educativos por tipo de empresa y actividad económica ${ }^{8}$

\begin{tabular}{|c|c|c|c|c|c|c|}
\hline \multirow{2}{*}{ Actividad económica } & \multirow{2}{*}{$\begin{array}{l}\text { Tipo de } \\
\text { empresa }\end{array}$} & \multicolumn{4}{|c|}{ Nivel educativo } & \multirow{2}{*}{ Total } \\
\hline & & 1 & 2 & 3 & 4 & \\
\hline \multirow{3}{*}{$\begin{array}{l}\text { A. Agricultura, ganadería, silvicultura y } \\
\text { pesca }\end{array}$} & Coop. & $48,0 \%$ & $44,1 \%$ & $5,8 \%$ & $2,2 \%$ & $100,0 \%$ \\
\hline & Merc. & $48,8 \%$ & $43,7 \%$ & $6,0 \%$ & $1,5 \%$ & $100,0 \%$ \\
\hline & Total & $48,6 \%$ & $43,8 \%$ & $6,0 \%$ & $1,6 \%$ & $100,0 \%$ \\
\hline \multirow{3}{*}{$\begin{array}{l}\text { BCDE. Industria extractiva y } \\
\text { manufacturera; suministro de energía } \\
\text { eléctrica, gas, vapor y aire } \\
\text { acondicionado; suministro de agua, } \\
\text { actividades de saneamiento, gestión de } \\
\text { residuos y descontaminación }\end{array}$} & Coop. & $21,3 \%$ & $53,7 \%$ & $15,8 \%$ & $9,2 \%$ & $100,0 \%$ \\
\hline & Merc. & $21,2 \%$ & $50,1 \%$ & $20,6 \%$ & $8,1 \%$ & $100,0 \%$ \\
\hline & Total & $21,2 \%$ & $50,3 \%$ & $20,4 \%$ & $8,1 \%$ & $100,0 \%$ \\
\hline \multirow{3}{*}{ F. Construcción } & Coop. & $24,4 \%$ & $66,9 \%$ & $7,1 \%$ & $1,6 \%$ & $100,0 \%$ \\
\hline & Merc. & $29,6 \%$ & $51,9 \%$ & $13,1 \%$ & $5,4 \%$ & $100,0 \%$ \\
\hline & Total & $29,6 \%$ & $52,1 \%$ & $13,0 \%$ & $5,3 \%$ & $100,0 \%$ \\
\hline \multirow{3}{*}{$\begin{array}{l}\text { G. Comercio al por mayor y por menor; } \\
\text { reparación de vehículos de motor y } \\
\text { motocicletas }\end{array}$} & Coop. & $28,0 \%$ & $51,8 \%$ & $13,2 \%$ & $7,0 \%$ & $100,0 \%$ \\
\hline & Merc. & $17,1 \%$ & $47,1 \%$ & $25,9 \%$ & $9,9 \%$ & $100,0 \%$ \\
\hline & Total & $17,9 \%$ & $47,5 \%$ & $24,9 \%$ & $9,7 \%$ & $100,0 \%$ \\
\hline \multirow{3}{*}{$\begin{array}{l}\text { HIJ. Transporte y almacenamiento; } \\
\text { hostelería; } \\
\text { comunicaciones }\end{array}$} & Coop. & $21,8 \%$ & $38,5 \%$ & $25,5 \%$ & $14,2 \%$ & $100,0 \%$ \\
\hline & Merc. & $20,3 \%$ & $48,5 \%$ & $22,3 \%$ & $8,9 \%$ & $100,0 \%$ \\
\hline & Total & $20,3 \%$ & $48,5 \%$ & $22,3 \%$ & $8,9 \%$ & $100,0 \%$ \\
\hline \multirow{3}{*}{ K. Actividades financieras y de seguros } & Coop. & $3,5 \%$ & $6,4 \%$ & $39,3 \%$ & $50,9 \%$ & $100,0 \%$ \\
\hline & Merc. & $4,4 \%$ & $10,7 \%$ & $42,7 \%$ & $42,1 \%$ & $100,0 \%$ \\
\hline & Total & $4,3 \%$ & $10,3 \%$ & $42,4 \%$ & $43,0 \%$ & $100,0 \%$ \\
\hline \multirow{3}{*}{$\begin{array}{l}\text { MN. Actividades profesionales, } \\
\text { científicas y técnicas; actividades } \\
\text { administrativas y servicios auxiliares }\end{array}$} & Coop. & $18,5 \%$ & $35,6 \%$ & $23,9 \%$ & $22,0 \%$ & $100,0 \%$ \\
\hline & Merc. & $24,9 \%$ & $41,7 \%$ & $22,2 \%$ & $11,2 \%$ & $100,0 \%$ \\
\hline & Total & $24,8 \%$ & $41,7 \%$ & $22,3 \%$ & $11,2 \%$ & $100,0 \%$ \\
\hline \multirow{3}{*}{ P. Educación } & Coop. & $6,5 \%$ & $13,1 \%$ & $21,1 \%$ & $59,3 \%$ & $100,0 \%$ \\
\hline & Merc. & $10,0 \%$ & $17,9 \%$ & $29,1 \%$ & $43,1 \%$ & $100,0 \%$ \\
\hline & Total & $9,7 \%$ & $17,5 \%$ & $28,5 \%$ & $44,3 \%$ & $100,0 \%$ \\
\hline \multirow{3}{*}{$\begin{array}{l}\text { QR. Actividades sanitarias y de } \\
\text { servicios sociales; actividades artísticas, } \\
\text { recreativas y de entretenimiento }\end{array}$} & Coop. & $8,3 \%$ & $52,1 \%$ & $20,9 \%$ & $18,7 \%$ & $100,0 \%$ \\
\hline & Merc. & $13,1 \%$ & $41,5 \%$ & $27,0 \%$ & $18,4 \%$ & $100,0 \%$ \\
\hline & Total & $12,9 \%$ & $42,0 \%$ & $26,7 \%$ & $18,4 \%$ & $100,0 \%$ \\
\hline \multirow{3}{*}{ TOTAL } & Coop. & $32,9 \%$ & $45,0 \%$ & $12,3 \%$ & $9,8 \%$ & $100,0 \%$ \\
\hline & Merc. & $23,9 \%$ & $45,1 \%$ & $20,8 \%$ & $10,3 \%$ & $100,0 \%$ \\
\hline & Total & $24,3 \%$ & $45,0 \%$ & $20,4 \%$ & $10,3 \%$ & $100,0 \%$ \\
\hline
\end{tabular}

Fuente: Elaboración propia a partir de la MCVL 2015

8 Calculado a partir del número de afiliaciones. No se han considerado las secciones de actividad para las que el número de afiliados en cooperativas es cero. 
Tabla. 12. Test chi-cuadrado para nivel educativo y tipo de organización para cada sección de actividad

\begin{tabular}{|c|c|c|c|}
\hline Actividad & $\begin{array}{l}\text { Valor chi- } \\
\text { cuadrado } \\
\left(\chi^{2}\right)\end{array}$ & $\begin{array}{l}\text { Grados } \\
\text { de } \\
\text { libertad }\end{array}$ & $\begin{array}{l}\text { Sig. } \\
\text { asintótica } \\
(2 \text { caras })\end{array}$ \\
\hline A. Agricultura, ganadería, silvicultura y pesca & 7,493 & 3 & 0,058 \\
\hline $\begin{array}{l}\text { BCDE. Industria extractiva y manufacturera; } \\
\text { suministro de energía eléctrica, gas, vapor y aire } \\
\text { acondicionado; suministro de agua, actividades de } \\
\text { saneamiento, gestión de residuos y } \\
\text { descontaminación }\end{array}$ & 9,342 & 3 & 0,025 \\
\hline F. Construcción & 13,503 & 3 & 0,004 \\
\hline $\begin{array}{l}\text { G. Comercio al por mayor y por menor; reparación } \\
\text { de vehículos de motor y motocicletas }\end{array}$ & 199,386 & 3 & 0,000 \\
\hline $\begin{array}{l}\text { HIJ. Transporte y almacenamiento; hostelería; } \\
\text { información y comunicaciones }\end{array}$ & 13,909 & 3 & 0,003 \\
\hline K. Actividades financieras y de seguros & 6,471 & 3 & 0,091 \\
\hline $\begin{array}{l}\text { MN. Actividades profesionales, científicas y } \\
\text { técnicas; actividades administrativas y servicios } \\
\text { auxiliares }\end{array}$ & 26,520 & 3 & 0,000 \\
\hline P. Educación & 27,213 & 3 & 0,000 \\
\hline $\begin{array}{l}\text { QR. Actividades sanitarias y de servicios sociales; } \\
\text { actividades artísticas, recreativas y de } \\
\text { entretenimiento }\end{array}$ & 23,679 & 3 & 0,000 \\
\hline Total & 428,102 & 3 & 0,000 \\
\hline
\end{tabular}

Fuente: Elaboración propia a partir de la MCVL 2015

Los resultados obtenidos indican que el nivel educativo y el tipo de organización empresarial, en este caso cooperativa y empresa mercantil, no están relacionados ni para el sector primario ni para el de actividades financieras y de seguros ya que los p-valores asociados han sido superiores a 0,05, es decir, las distribuciones son homogéneas y no existen diferencias significativas en el nivel educativo por pertenecer a una u otra organización; no obstante, hay que indicar que dichos valores se encuentran en el límite ya que los p-valores están cercanos a éste. Para el resto de actividades económicas, sí existe relación entre el nivel educativo y la pertenencia a cooperativa o empresa mercantil (ya que los p-valores asociados han resultado inferiores a 0,05 , rechazándose la hipótesis nula de independencia entre las variables analizadas) (Tabla 12).

Las sociedades cooperativas superan en porcentaje de empleados con un nivel educativo elevado (títulos universitarios y de postgrado) a las empresas mercantiles o convencionales en las siguientes actividades según la clasificación realizada (Tabla 11): industria en general; transporte y almacenamiento, hostelería, información y comunicaciones; actividades financieras y de seguros; actividades profesionales, científicas y técnicas; actividades administrativas y servicios 
auxiliares y educación. Esto plantea la necesidad de resaltar que, la ES en general y las cooperativas en particular, no deben considerarse como entidades de carácter subsidiario en los sistemas económicos actuales, como se ha hecho tradicionalmente, ya que, aunque todavía no puede hablarse de altas cifras, el personal que desarrolla las actividades descritas cuentan con niveles educativos elevados.

De lo anterior se deduce que para aquellas actividades que requieren de capital humano cualificado y de adaptación a cambios tecnológicos que se traduzcan en mayor eficiencia en los servicios a empresas que los reciben como insumos, como son los servicios avanzados y auxiliares a empresas, las cooperativas tienen un porcentaje de trabajadores con titulaciones universitarias y de postgrado (22\%) superior al de empresas mercantiles $(11,2 \%)$. Por tanto, a pesar de que no sea una actividad de un peso relativo significativo dentro del cooperativismo andaluz, como se ha descrito anteriormente, cuenta con una notable formación de los trabajadores. Por su parte, las empresas mercantiles presentan porcentajes superiores para este nivel educativo tan solo en la construcción y en el comercio al por mayor y por menor, reparación de vehículos de motor y motocicletas.

En cuanto a los niveles educativos bajos, la diferencia más significativa se produce en el sector de la construcción para el que el $29,6 \%$ de los afiliados a empresas mercantiles no tiene titulación o su nivel es inferior al de graduado escolar, mientras que esta proporción es del $24 \%$ para las cooperativas.

En términos generales, atendiendo al total de afiliaciones sin diferenciar el sector económico, la proporción de trabajadores con nivel educativo básico (nivel 2) y superior (nivel 4) para las cooperativas y para las empresas mercantiles es aproximadamente la misma, existiendo diferencias en el resto de niveles. En esta línea, hay que destacar que, para el nivel educativo más bajo (nivel 1), la proporción de trabajadores en cooperativas es más elevada que en el sector empresarial tradicional (alrededor del 33\% frente a un $24 \%$ respectivamente) (Tabla 11).

\section{Consideraciones finales}

El principal objetivo del presente trabajo ha sido determinar el perfil del empleo en las sociedades cooperativas y obtener una aproximación a la calidad del mismo respecto al sector empresarial tradicional o mercantil, representado éste por las sociedades anónimas y sociedades limitadas, para el caso de Andalucía. El empleo ha sido analizado desde el punto de vista del tipo de contrato (indefinido o temporal), de la jornada laboral, de las secciones de actividad, del nivel educativo y del grupo de cotización, desde diferentes perspectivas, a partir de distintos colectivos sociales implicados (empleo de mujeres frente al de hombres y empleo por edades).

Comenzando por la estabilidad en el empleo, en concreto por el número de afiliaciones medias por trabajador, hay que destacar que este número es menor en las cooperativas $(1,66)$ que en las sociedades mercantiles $(2,10)$, lo que indica cierta estabilidad laboral superior en las primeras, al menos en cuanto a la duración de los contratos ya que, en lo que respecta al tipo de contrato, indefinido o 
temporal, la cifra de temporalidad es ligeramente superior en las cooperativas que en el sector empresarial tradicional: 3,68 y 3 contratos temporales por cada indefinido respectivamente; esto se debe principalmente a que una gran proporción de trabajadores en cooperativas pertenecen al sector agrario el cual está afectado por la propia estacionalidad de sus actividades.

Desde una perspectiva de género se puede sostener que las cooperativas generan mayores oportunidades laborales para las mujeres que las sociedades mercantiles. Esto se ha podido concluir a partir de los resultados que se recogen a continuación:

En primer lugar, en las cooperativas existe un mayor número de mujeres que de hombres, sobre todo destacando una mayor presencia de este empleo femenino en los contratos temporales, ya que para los indefinidos no existen diferencias significativas entre ambos; por su parte, para el caso de las sociedades mercantiles, el número de hombres es mayor que el de mujeres tanto en los contratos indefinidos como en los temporales, con diferencias significativas. Por tanto, las mujeres representan un mayor número en las cooperativas que en las empresas mercantiles, destacando su mayor presencia en puestos más elevados -con mayor capacidad de decisión- en mayor grado que en el sector empresarial tradicional.

En segundo lugar, hay que destacar que atendiendo al grupo de cotización, para los más elevados y que requieren de ciertas cualificaciones como la capacidad directiva y de liderazgo empresarial, específicamente para los de ingenieros, licenciados y alta dirección; ingenieros técnicos, peritos y ayudantes; y jefes administrativos y de taller, el género no está relacionado con el tipo de contrato indefinido o temporal, en las cooperativas, como han demostrado los test de Yates; es decir, no existen diferencias para estos grupos entre hombres y mujeres por tipo de contrato. Tampoco existen diferencias para los grupos de cotización de oficiales administrativos, de oficiales de tercera y especialistas y de mayores de 18 años no cualificados. Asimismo, debe señalarse que para el resto de grupos de cotización, en los que el sexo sí influye en el tipo de contrato, siendo éstos tan solo cuatro de los diez considerados, estas diferencias se deben al número superior de mujeres que de hombres en valores absolutos, en mayor medida en los contratos temporales. Por tanto, a nivel general hay que destacar que no existen diferencias significativas de género por tipo de contrato en las cooperativas, acentuando que, en los grupos de cotización donde sí existen diferencias, éstas se deben a la mayor presencia del colectivo femenino.

Para las sociedades mercantiles la situación es muy diferente. En el sector empresarial tradicional, a nivel general, el número de hombres es significativamente superior que el de mujeres, representando un $58 \%$ y $42 \%$ respectivamente. A diferencia de lo que sucede en las sociedades cooperativas, para los grupos de cotización más elevados, el test de Yates ha confirmado que la variable sexo sí influye en el tipo de contrato (indefinido o temporal), debiéndose esto al mayor número de hombres que de mujeres. Por otro lado, no existe relación entre estas variables para los grupos de oficiales administrativos, subalternos y oficiales de primera y de segunda.

En tercer y último lugar, en lo que concierne al perfil del empleo en cooperativas desde la perspectiva de género, cabe señalar la mayor proporción de mujeres que de hombres con jornadas a tiempo parcial tanto en las cooperativas como en las sociedades mercantiles, existiendo asociación entre el sexo y la 
jornada laboral (los test de corrección de Yates han sido significativos a un nivel de confianza del 99\%). Si se hace una comparación de la proporción de mujeres a jornada parcial sobre el total de este colectivo, esta proporción es bastante menor en las cooperativas, lo que significa que -aunque no sea determinante para medir la calidad en el empleo puesto que se desconoce si la jornada ha sido por elección propia o no- las mujeres cuentan con mayores oportunidades en las cooperativas si quieren trabajar a tiempo completo.

Pero estas ventajas de las sociedades cooperativas como organizaciones más significativas de la ES, no se han puesto de manifiesto únicamente en el colectivo femenino: en cooperativas, la proporción de trabajadores menores de 30 años (24\%) y la de mayores de 45 (31\%) son ligeramente superiores a la de empresas mercantiles ( $23 \%$ y $29 \%$ respectivamente).

Tanto los menores de 30 años y los mayores de 45 como el colectivo femenino presentan en la actualidad importantes dificultades para incorporarse al mercado laboral o lograr puestos de trabajo estables. Frente a estas dificultades, las sociedades cooperativas pueden presentarse como oportunidad o alternativa para paliar dichos impedimentos en mayor medida que el sector tradicional empresarial, poniendo en evidencia la responsabilidad y compromiso con estos colectivos susceptibles de padecer mayores riesgos de exclusión laboral. La mayor proporción de estos trabajadores en cooperativas que en empresas mercantiles, podría derivarse de la generación de emprendimientos colectivos como alternativa a la situación de desempleo.

En lo que concierne a las actividades con mayor peso del empleo hacia las que se dirige el cooperativismo, se puede confirmar la fuerte presencia de éste en el sector primario, de considerable peso en la región andaluza. Se trata de un empleo con un marcado carácter temporal (ya que el $96 \%$ de los trabajadores del sector tienen contratos temporales), representando éstos a su vez -es decir, solo los temporales del sector primario- un $40 \%$ del total del empleo generado por las sociedades cooperativas. Estas cifras son notoriamente más bajas en el sector empresarial tradicional ya que, aunque la proporción de trabajadores temporales dentro de este sector es también elevado (un 94\%), dicho sector solo representa el $11 \%$ del total del empleo generado en estas sociedades mercantiles.

No obstante, hay que indicar que la temporalidad en el sector agrario es un problema estructural en Andalucía. En la década de los 80 tuvieron lugar una serie de acontecimientos -como la incorporación a la Unión Europea, emigraciones desde las áreas rurales, la reforma agraria, las reivindicaciones por parte de campesinos para mejorar las condiciones laborales o las diferencias económicas con el resto de regiones- que llevaron a la aparición del subsidio de desempleo agrario en 1984 y la posterior renta agraria en 2002 (Cejudo, Navarro y Maroto, 2016). Hay quienes afirman que este subsidio agrario ha generado desempleo estructural por desalentar la búsqueda activa de empleo y por cierto hábito de la "cultura del subsidio" dadas las facilidades de acceder a estas protecciones sociales, a lo que hay que añadir un determinado efecto de marginación por parte de los empresarios hacia los desempleados de larga duración (Murillo, Núñez y Usabiaga, 2005: 47).

Además, este efecto de desempleo de larga duración afecta de manera negativa al capital humano (Murillo, Núñez y Usabiaga, 2005), limitando y mermando las 
capacidades de formación y cualificación de estos trabajadores. En línea con esta idea, alrededor de la mitad de los trabajadores del sector agrario analizados en este trabajo (tanto de cooperativas como de empresas mercantiles) no tienen titulación alguna o tiene un nivel inferior al de graduado escolar, lo que confirma lo anterior. Esto crea un círculo vicioso en torno al desempleo de carácter estructural del sector agrario, ya que estos trabajadores no están formados y a su vez o no tienen oportunidad o predisposición a ello, según se trate, para incorporarse a puestos de trabajo que hagan progresar en la mejora de sus capacidades, de su capital humano. No obstante, habría que determinar qué tipo de temporalidad se debe a la propia estacionalidad del sector y cual al carácter de eventualidad, ya que son estas últimas las que generan los mayores efectos negativos sobre la formación del capital humano y la reincorporación a otros puestos de trabajo.

Continuando con los resultados derivados del empleo generado según secciones de actividad, hay que destacar una mayor presencia de éste en las sociedades cooperativas, en comparación con el sector empresarial tradicional, en las actividades educativas y sanitarias y de servicios sociales. Esto pone en evidencia los principios y valores sociales bajo los que trabajan las organizaciones de ES.

Por otro lado, el peso del cooperativismo, en términos de empleo, es de menor relevancia para las actividades de servicios auxiliares a empresas y actividades profesionales, científicas y técnicas, que en las empresas mercantiles. En esta línea, no obstante, debe señalarse que el nivel educativo de los trabajadores para estas actividades es superior que el de las empresas mercantiles: los empleados con estudios universitarios y de postgrado representan el $22 \%$ sobre el total en estas actividades para las cooperativas, mientras que para las sociedades mercantiles este porcentaje se reduce a la cifra de $11,2 \%$. Esto induce a la idea de que existe un capital humano significativo en las sociedades cooperativas dedicadas a estas actividades a pesar de que, cuantitativamente, no generen un empleo considerable en relación a otras actividades como puede ser el sector agrario. Este mayor nivel educativo supone un potencial de capital humano para fomentar el desarrollo del cooperativismo que debería ser canalizado mediante medidas y estrategias de carácter público y privado.

No obstante, por otro lado y como una de las limitaciones del presente trabajo, se hace necesario indicar que un nivel educativo elevado no garantiza el aprovechamiento de este potencial por parte de las organizaciones ya que pueden darse situaciones de subempleo por la no correspondencia entre la formación y el puesto en la empresa que ocupe un trabajador; determinar dicha correspondencia se plantea, de esta forma, como futura línea de investigación.

Por otro lado, se ha realizado un análisis de carácter estático, para un determinado momento del tiempo (año 2015), que supone una limitación para analizar la dinámica evolutiva que ha seguido el perfil y la calidad del empleo de las cooperativas. De ahí que se establezca también, como futura línea de investigación, identificar la trayectoria del empleo generado por estas sociedades de economía social, en lo que a calidad del empleo se refiere.

Como última línea de investigación futura que surge del presente trabajo, se establece analizar en mayor profundidad, por la importancia relativa que suponen y por los resultados aquí obtenidos, el comportamiento del empleo que generan las sociedades cooperativas andaluzas del sector primario. 
Finalmente, hay que destacar que, a pesar de estas limitaciones, las ventajas que suponen las cooperativas en términos de empleo para determinados colectivos sociales, como mujeres y jóvenes, se convierte en un potencial y justificación para impulsar el desarrollo de estas organizaciones en el contexto socioeconómico actual.

Este trabajo supone, por un lado, la justificación de fomentar el cooperativismo en Andalucía y, por otro, un mejor conocimiento del empleo generado por las cooperativas de la región, que debe tenerse en cuenta en el diseño de estrategias y políticas específicas encaminadas a fomentar el empleo.

\section{Referencias bibliográficas}

Alianza Cooperativa Internacional (ACI) (1995) ¿Qué es una cooperativa? Principios y Valores Cooperativos. Disponible en: http://ica.coop/es/node/10584 (Consultado el $8 / 12 / 2016)$.

Barea, J. (2008) El concepto científico de economía social desde la perspectiva actual. Temas para el Debate, $\mathrm{N}^{\circ} 167$, Octubre, ISSN: 1134-6574.

Calderón, B. y Calderón, M.J. (2012a) Cómo afrontan la crisis las cooperativas en España: comparativa de trayectorias laborales a partir de la Muestra Continua de Vidas Laborales. Ciriec-España. Revista de Economía Pública, Social y Cooperativa, $\mathrm{N}^{\mathrm{0}} 76$, pp. 5-26.

Calderón, B., y Calderón, M. J. (2012b) La calidad del empleo de las entidades de la economía social en período de crisis. EKONOMIAZ, Revista vasca de Economía, № 79, Vol. (01), pp. 31-58.

Cebrián, I. y Toharia, L. (2008) La entrada en el mercado de trabajo. Un análisis basado en la MCVL. Revista de Economía Aplicada, № 16, Vol. 1, pp.137-172.

CECOP-CICOPA Europa. (2011) A pesar de las dificultades, las cooperativas de industria y servicios, resisten a la crisis y sus consecuencias. Tercer informe anual sobre la crisis, Junio 2011.

Cejudo, E., Navarro, F.A. y Maroto, J. C. (2016) Mercados de Trabajo y Prestaciones Asistenciales Agrarias en las Regiones Españolas de Andalucía y Extremadura: diferencias territoriales y perfiles demográficos de sus beneficiarios. Brazilian Journal of Rural Economy and Sociology (RESR), № 54, Vol. 2, pp. 339-360.

Clemente, J., Díaz-Foncea, M., Marcuello, C. y Sanso-Navarro, M. (2012) The wage gap between cooperative and capitalist firms: evidence from Spain. Annals of Public and Cooperative Economics, № 83, Vol. 3, pp. 337-356.

Clemente, J., García, I. y Sanso, M. (2008) Análisis de las diferencias salariales entre trabajadores indefinidos. Revista de Economía Aplicada, $\mathrm{N}^{\circ}$ 16, Vol. 1, pp. 93-135.

Comisión de las Comunidades Europeas. (2001) Políticas sociales y de empleo: un marco para invertir en la calidad. Comunicación de la Comisión al Consejo, al Parlamento Europeo, al CESE y al Comité de las Regiones, de 20 de junio de 2001 [COM (2001) 313 final].

Cornforth, C. y Thomas, A. (1995) Les cooperatives de travail au Royaume Uni. Declin ou croissance? Revue des Etudes Cooperatives, Mutualistee et Associatives, № 255, Vol. 53.

Díaz, M. y Marcuello, C. (2010) Impacto económico de las cooperativas. La generación de empleo en las sociedades cooperativas y su relación con el PIB. Ciriec-España. Revista de Economía Pública, Social y Cooperativa, No 67, pp. 23-44. 
Divar, J. (2011) Las cooperativas: una alternativa económica. Madrid: Dykinson.

Domínguez-Fabián, I. y Encinas-Goenechea, B. (2008) Inmigración y solvencia financiera del sistema público de pensiones tras la regularización de 2005. Revista de Economía Aplicada, No 16, Vol. 1, pp. 67-92.

García-Gutiérrez, C., Lejarriaga, G., Bel, P., Fernández, J. y Martín, S. (2013) Empresas individuales y trabajo autónomo: evolución y previsiones. En G. Lejarriaga, S. Martín y A. Muñoz (dirs.), 40 años de historia de las Empresas de Participación. Madrid: editorial Verbum, pp. 342-366.

Grávalos, M.A., Pomares, I. (2001) Cooperativas, desempleo y efecto refugio. REVESCO. Revista de Estudios Cooperativos, $\mathrm{N}^{\mathrm{0}} 74$, pp. 69-84.

Guerreri, G., Nazzaro, O. y Zevi, A. (1992) La economía social en Italia. En J.L. Monzón y J. Defourny (Dirs.), Economía Social, entre economía capitalista y economía pública. Valencia: CIRIEC-España editorial y Ministerio de Trabajo y Seguridad Social, pp. 157-198.

Lejarriaga, G., Bel, P. y Martín, S. (2013) El emprendimiento colectivo como salida laboral de los jóvenes: análisis del caso de las empresas de trabajo asociado. REVESCO. Revista de Estudios Cooperativos, $\mathrm{N}^{\mathrm{o}} 112$, pp. 36-65. DOI: 10.5209/rev_REVE.2013.v112.43068.

Martínez, I.M, Arcas, N. y García, M. (2011) La influencia del género sobre la responsabilidad empresarial en las entidades de Economía Social. REVESCO. Revista de Estudios Cooperativos, Segundo Cuatrimestre, $\mathrm{N}^{\mathrm{o}} 105$, pp. 143-172. DOI: 10.5209/rev_REVE.2011.v105.6.

Monzón, J.L. (2006) Economía Social y conceptos afines: fronteras borrosas y ambigüedades conceptuales del Tercer Sector. Ciriec-España. Revista de Economía Pública, Social y Cooperativa, No 56, pp. 9-24.

Moral-Arce, I., Patxot, C. y Souto, G. (2008) La sostenibilidad del sistema de pensiones. Una aproximación a partir de la MCVL. Revista de economía aplicada, № 16, Vol. 1, pp. 29-66.

Murillo, I., Núñez, F. y Usabiaga, C. (2005) Análisis del Diferencial de Desempleo Andaluz y Extremeño. Revista de Estudios Regionales, № 72, pp. 45-83.

Santero, R., Castro, B. (2016) Análisis de las condiciones laborales en las entidades de la Economía Social en España desde una perspectiva de género. REVESCO. Revista de Estudios Cooperativos, Segundo Cuatrimestre, No 121, pp. 228-255. DOI: 10.5209/rev_REVE.2016.v121.51309.

Tomas-Carpi, J.A. (1997) Prospects for a Social Economy in a changing world. Annals of Public and Cooperative Economics, $\mathrm{N}^{\circ}$ 68, Vol. 2, pp. 247-279. 